\title{
Validation of a Method for Simultaneous Isolation of Shiga Toxin-Producing Escherichia coli O26, O103, 0111, and 0145 from Minced Beef by an International Ring-Trial
}

\author{
Karen Verstraete, Lieven De Zutter, Joris Robyn, Georges Daube, ${ }^{3}$ Lieve Herman, \\ Marc Heyndrickx, ${ }^{1,4}$ Marie-Athénaïs de Schaetzen, ${ }^{3}$ and Koen De Reu ${ }^{1}$
}

\begin{abstract}
An isolation method described by Possé et al. (FEMS Microbiol Lett 2008;282:124-131) was satisfactorily validated in an international ring-trial using artificially contaminated minced beef samples. Until now, no validated method existed for the simultaneous isolation of Shiga toxin-producing Escherichia coli serogroups O26, O103, O111, and $\mathrm{O} 145$ in food. Twelve laboratories from five European countries participated and received 16 inoculated beef samples contaminated with cold-stressed cells of the four serogroups O26, O103, O111, and O145 in two levels (approximately 30 and $300 \mathrm{CFU} 25 \mathrm{~g}^{-1}$ ) in duplicate. In addition, they received four non-inoculated samples. The isolation protocol comprised a selective enrichment step, a selective isolation step on a non-O157 agar plate differentiating the serogroups by color, followed by confirmation by plating on confirmation agar media and agglutination. All laboratories were able to isolate the inoculated serogroups from the samples, both for the high and the low inoculation level. Results did not differ whether in-house-prepared or ready-to-use nonO157 agar plates were used, demonstrating that by following the instructions laboratories managed to perform the complete protocol with success.
\end{abstract}

\section{Introduction}

$S^{\prime}$ HIGA TOXIN-PRODUCING Escherichia coli (STEC) are a very important emerging group of foodborne pathogens, capable of causing the life-threatening complication hemolytic uremic syndrome (HUS). The majority of cases worldwide are caused by strains belonging to the serogroup O157, but infections caused by other serogroups (non-O157) have increasingly been reported (Bettelheim, 2007; Ethelberg et al., 2009; Schimmer et al., 2008). According to a European Food Safety Authority (EFSA) report, the major serotypes of concern are E. coli O157:H7, O26, O91, O103, O111, and O145. Bovine beef is believed to be a major source of foodborne STEC infections for humans (EFSA, 2011). Prevalence data of STEC in bovine minced beef were $2.3 \%$ in Europe in 2009 (EFSA, 2011).

A standardized isolation method is available for serogroup O157, based on specific properties of E. coli O157 strains, like the inability to ferment sorbitol, and the resistance to bile salts, crystal violet, cefixime, and tellurite. Currently, for non-O157 STEC, no international standard method for isolation is available, mainly because of the lack of common phenotypic characteristics. At present, a Working Group (CEN TC275/ WG6) has drafted and submitted to International Organisation for Standardization (ISO) a real-time polymerase chain reaction (PCR)-based horizontal method for the detection of STEC belonging to O26, O103, O111, O145, and O157, including sorbitol fermenting (SF) STEC O157 serogroups in food (EFSA, 2009). However, positive samples still need confirmation by the isolation of the STEC strain, which remains a difficult challenge. For that, a non-selective E. coli medium (TBX, a tryptone-bile-glucuronic medium) was recommended for screening of colonies at random by PCR for the presence of the virulence genes. This is labor intensive, and colonies harboring the combination of the detected genes are frequently not found. However, the Working Group also

Collaborators: F. Auvray, J. Boel, N. Botteldoorn, M. Cudjoe, G. Daube, K. De Reu, L. De Zutter, A. Heuvelink, D. Piérard, M. Rannou, C. Rozand, and M. Uyttendaele.

${ }^{1}$ Technology and Food Science Unit, Institute for Agricultural and Fisheries Research, Melle, Belgium.

Departments of ${ }^{2}$ Veterinary Health and Food Safety and ${ }^{4}$ Pathology, Bacteriology and Poultry Diseases, Ghent University, Merelbeke, Belgium.

${ }^{3}$ Food Science Department, Faculty of Veterinary Medicine, University of Liège, Liège, Belgium. 
proposed the use of selective media such as the non-O157 agar described by Possé et al. (2008a).

Our research group focused on the development of a method for the isolation of the four clinically important nonO157 STEC serogroups belonging to "the gang of five," namely STEC O26, O103, O111, and O145 (Beutin, 2006) from food and feces. Serogroup O91 was not included in the development of this method, as the incidence of HUS associated with this serogroup is low (Karmali et al., 2003). An enrichment broth, a non-O157 agar, and confirmation agars for the biochemical confirmation of each of the serogroups were developed for these four STEC serogroups by Possé et al. (2008b). The non-O157 agar plates are based on a mixture of carbohydrate sources and a chromogenic substrate for $\beta$-Dgalactosidase, whereas the confirmation agars contain one specific carbohydrate source each. This isolation method was evaluated in a intralaboratory study for various food matrices and was found effective at recovering low numbers (4-36 CFU $25 \mathrm{~g}^{-1}$ ) of cold-stressed and non-stressed STEC serogroups (O26, O103, O111, and O145) from artificially contaminated minced beef and raw-milk cheese (Verstraete $e t$ al., 2012). The aim of the present study was to validate the isolation method by an international ring-trial as the next step in the validation procedure. Minced beef samples were artificially contaminated with STEC O26, O103, O111, and O145, and analyzed by 12 participating laboratories in Europe.

\section{Methods}

\section{Design of the trial}

Twelve European laboratories from five countries (Belgium, the Netherlands, Denmark, France, and Norway) participated in the ring-trial. All laboratories had experience in the isolation of E. coli $\mathrm{O} 157$ from food matrices. Six weeks before the ring-trial, a workshop with demonstrations was organized to inform the participants about the design of the trial and all aspects of the isolation method, with special attention given to the colony morphology of the different serogroups on the non-O157 agar. At the end of the workshop, all laboratory products to prepare enrichment and isolation media were provided to the participants to further practice in order to become familiar with the protocol. Two weeks before the ring-trial, all laboratories received the laboratory products to prepare all necessary media, including the in-house-prepared non-O157 agar plates and the final standard operating procedure (SOP). For the actual ring-trial, each laboratory received 18 blind-coded 25-g minced beef samples, from which 16 were inoculated with one of the four STEC serogroups (O26, O103, O111, and O145) in two levels (30 and 300 CFU $25 \mathrm{~g}^{-1}$ ) in duplicate; two were non-inoculated samples used as blank references. In addition, two non-inoculated samples were provided: one for temperature registration (sample T) and one for bacterial counts of background microbiota (sample C). A sufficient number of ready-to-use nonO157 agar plates, prepared in laboratory N (Ghent University, Department of Veterinary Health and Food Safety); the serological latex test kits for serogroups O26, O103, O111, and O145 (Statens Serum Institut, Copenhagen, Denmark); and a reporting sheet were sent together with the samples. Sample analysis was to start 2 days after shipment. The reporting sheet with the obtained results was to be returned by all laboratories. Participants were asked to detail all their re- marks and any additional information that could have influenced their results.

\section{Preparation of inocula}

Artificial inoculation was performed with STEC strains belonging to serogroup O26 (MB2674), O103 (MB2653), O111 (MB2678), or O145 (MB2676) originating from human clinical infections in Belgium and kindly donated by the Belgian national VTEC reference laboratory. Strains were stored at $-80^{\circ} \mathrm{C}$ using Pro-Lab Microbank cryovials (Pro-Lab, Ontario, Canada) according to the manufacturer's instructions and cultured on Tryptone Soy Agar (TSA; Oxoid, Ltd., London, $\mathrm{UK})$ at $37^{\circ} \mathrm{C}$ for $24 \mathrm{~h}$. From each culture plate, one colony was transferred into Tryptone Soy Broth (TSB; Oxoid) and incubated at $37^{\circ} \mathrm{C}$ for $24 \mathrm{~h}$. For stress treatment, $5 \mathrm{~mL}$ of the stationary phase cultures was kept at $4^{\circ} \mathrm{C}$ for 7 days (cold-stress). On day 6 of cold-stress, the number of E. coli cells was determined by plating 10-fold serial dilutions made in Buffered Peptone Water (BPW; Oxoid) on TSA in duplicate, and incubated at $37^{\circ} \mathrm{C}$ for $24 \mathrm{~h}$. Based on the enumeration, the appropriate volumes for inoculation were calculated. After 7 days of cold-stress, the cultures were 10-fold serially diluted in BPW (Oxoid) and used for artificial inoculation of subsamples. At the same time, the diluted cultures were plated on TSA (Oxoid) in duplicate and incubated at $37^{\circ} \mathrm{C}$ for $24 \mathrm{~h}$ to estimate the number of inoculated cells.

\section{Sample inoculation and shipment}

All samples originated from one batch of fresh minced beef, which was purchased from a local butcher in Ghent and which first tested negative for STEC O26, O103, O111, O145, O157 NSF, and O157 SF using the isolation method as described by Possé et al. (2008b). Twenty-five-gram subsamples were transferred to stomacher bags with filter. Based on the quantification of the cold-stressed STEC cultures, appropriate volumes of the $10^{-6}$ diluted bacterial culture were pipetted into the center of the samples, to obtain contamination levels of 30 CFU $25 \mathrm{~g}^{-1}$ and 300 CFU $25 \mathrm{~g}^{-1}$ minced beef for each single serogroup in duplicate. Packages were prepared to provide a complete set of 20 samples for each laboratory. A sufficient number of ready-to-use non-O157 agar plates and serological latex test kits (Statens Serum Institut) were included. Samples were packaged according to the European legislation UN2814 with addition of thermo ice-blocks and an insulating packaging to ensure cooled transportation. Sample packages were stored at $4^{\circ} \mathrm{C}$ for $3 \mathrm{~h}$ before shipment.

\section{Reception of samples and bacterial counts of background microbiota}

Upon receipt, each laboratory measured the temperature of the sample $\mathrm{T}$ using a temperature probe. This information together with the date of receipt of the samples was noted on the reporting sheet. Sample C was 1/10 serially diluted using TSB (Oxoid) and homogenized by stomaching for $2 \mathrm{~min}$. Numbers of aerobic mesophiles, Enterobacteriaceae, and E. coli were determined by plating on Petrifilm Aerobic Count Plates (3M, Maplewood, MN), Petrifilm Enterobacteriaceae Counts (3M), and Petrifilm E. coli/Coliform Count plates (3M), and incubated at $30^{\circ} \mathrm{C}, 37^{\circ} \mathrm{C}$, and $44^{\circ} \mathrm{C}$, respectively, for 18-24h. 


\section{STEC isolation procedure performed by the participating laboratories}

All other samples were $1 / 10$ diluted by adding $225 \mathrm{~mL}$ of TSB (Oxoid), subsequently homogenized by stomaching for two minutes and subjected to the isolation protocol based on Possé et al. (2008b) and described in the SOP. The protocol, which is presented in Figure 1, included a sample enrichment, a consecutive isolation on a non-O157 agar with differentiation of the different serogroups by color (Fig. 2) and confirmation on confirmation agar plates, followed by serology using agglutination. Briefly, $8 \mathrm{mg} \mathrm{L}^{-1}$ novobiocine (Sigma, St. Louis, MO) and $16 \mathrm{mg} \mathrm{L}^{-1}$ vancomycin (Sigma) were added to the TSB enrichment broth. After $6 \mathrm{~h}$ of a first enrichment step at $37^{\circ} \mathrm{C}, 2 \mathrm{mg} \mathrm{L}^{-1}$ rifampicin (Sigma), $1.5 \mathrm{~g}$ $\mathrm{L}^{-1}$ bile salts (Oxoid), and $1.0 \mathrm{mg} \mathrm{L}^{-1}$ potassium tellurite (Sigma) were added to the first enrichment broth to prepare the second enrichment broth, and incubation was continued at $42^{\circ} \mathrm{C}$ for $18 \mathrm{~h}$. Post-incubation, $100 \mu \mathrm{L}$ of the second enrichment broth was spread on the non-O157 agar plates using an inoculation loop, with subsequent incubation at $37^{\circ} \mathrm{C}$ for $24 \mathrm{~h}$. Colonies were evaluated based on their general appearance and color, and suspected colonies were streaked onto two of four specific confirmation agar plates according to the scheme given in Figure 1. Only if the color of the colony was in accordance to the scheme, the colony remained suspected. Finally, colonies were confirmed by serology using latex test kits for serogroups O26, O103, O111, and O145 (Statens Serum Institut).

\section{Statistical analysis}

The ring-trial results were analyzed statistically according to the recommendations of EN ISO 16140 (ISO, 2003). The diagnostic sensitivity was defined as the percentage of positive samples giving a correct positive signal. The diagnostic specificity was defined as the percentage of negative samples giving a correct negative signal. Accordance (repeatability of qualitative data) was defined as the percentage chance of finding the same result, positive or negative, from two identical samples analyzed in the same laboratory under predefined repeatability conditions. Concordance (reproducibility of qualitative data) was defined as the percentage chance of finding the same result, positive or negative, from two identical samples analyzed in different laboratories under predefined reproducibility conditions. These calculations take into account different replications in different laboratories by weighing results appropriately.

The bacterial counts of the background microbiota of the minced beef were log-transformed prior to calculations.

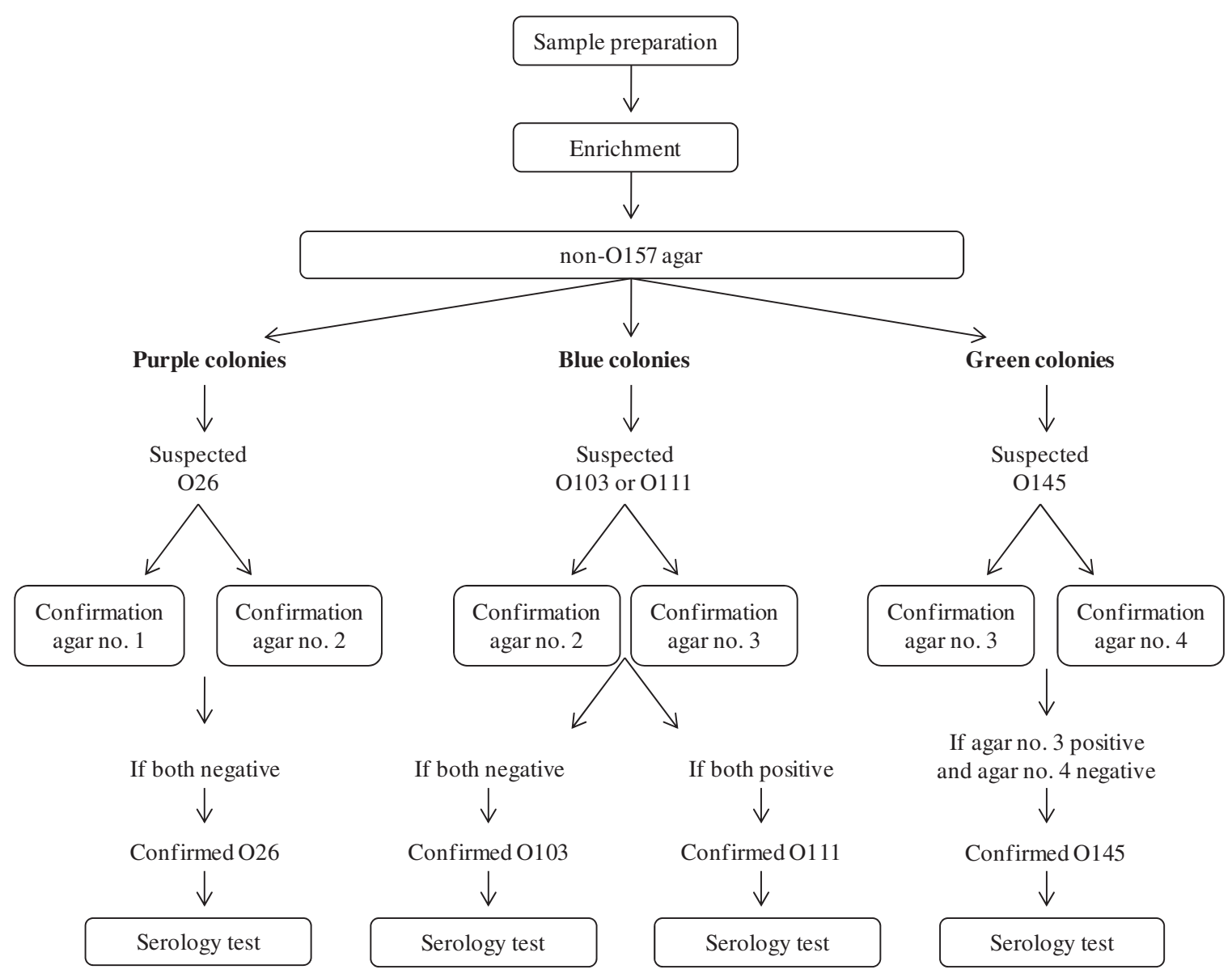

FIG. 1. Protocol applied by the participating laboratories of the international ring-trial for the isolation of Shiga toxinproducing Escherichia coli (STEC) O26, O103, O111, and O145 from minced beef, based on Possé et al. (2008b). Confirmation agar plate no. 1, containing L-rhamnose; no. 2, containing dulcitol; no. 3, containing D-arabinose; no. 4, containing D-raffinose. 


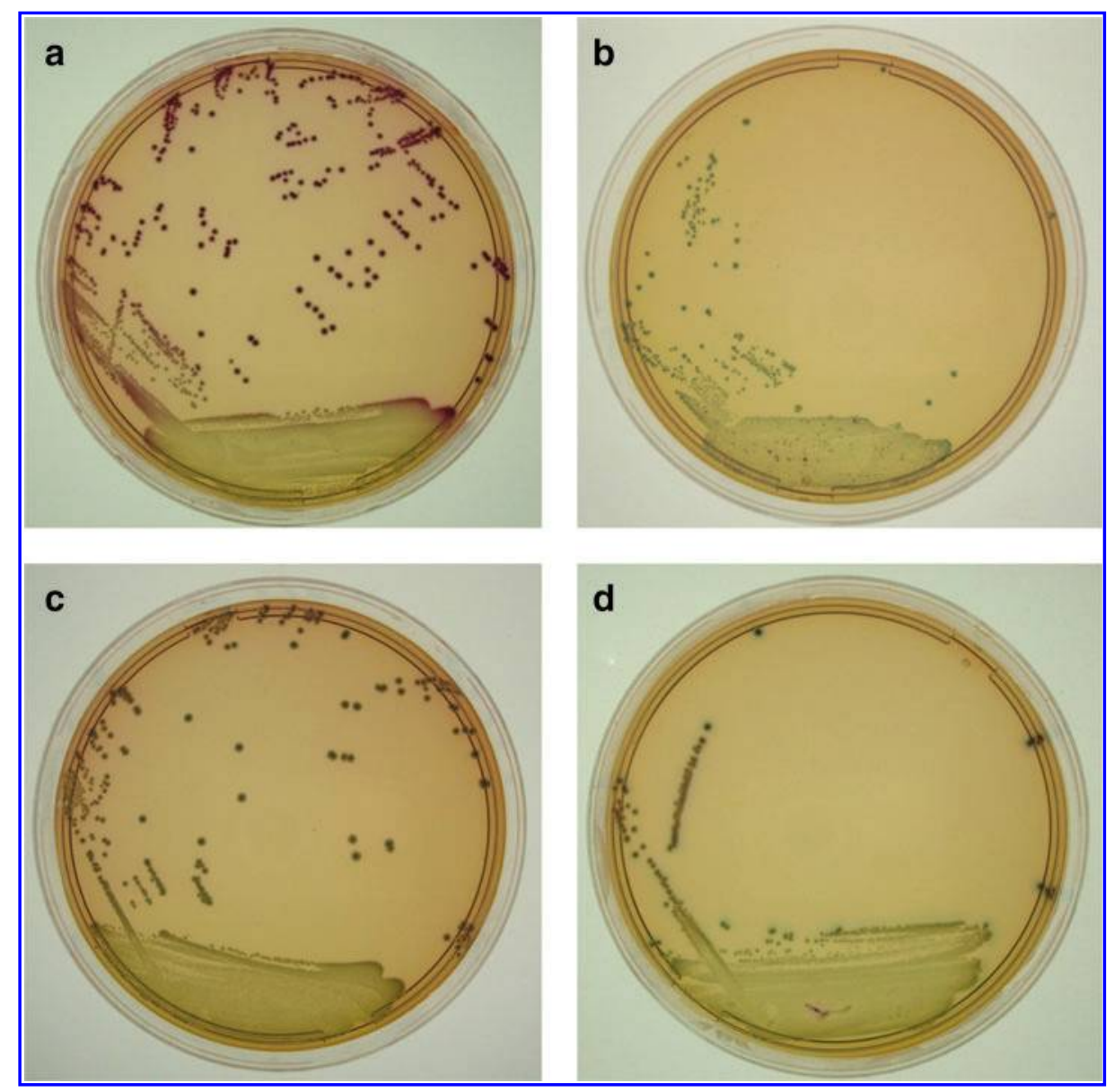

FIG. 2. Growth of Shiga toxin-producing Escherichia coli (STEC) cells and background microbiota on the non-O157 agar (Possé et al., 2008a) for the recovery of STEC serogroups O26 (a), O145 (b), O111 (c), and O103 (d) from artificially contaminated minced beef in an international ring-trial. STEC O26, O145, O111, and O103 are present as purple, green, blue, and blue colonies, respectively. The background microflora is not present as single colonies, but overgrown by STEC in the confluent zone.

\section{Results}

\section{Artificial inoculation}

The non-inoculated subsamples were found negative for the four STEC serogroups by all laboratories. Inoculation levels are detailed in Table 1 and ranged from 25-33 CFU $25 \mathrm{~g}^{-1}$ for the low level to $264-411$ CFU $25 \mathrm{~g}^{-1}$ for the high level.

\section{Temperature registration and bacterial counts of background flora at receipt}

Most laboratories received the samples on the same day of shipment (six laboratories), four laboratories on the next day, and only one laboratory each on the second or the third day after shipment. Ten of 12 laboratories registered sample temperatures of $\leq 5^{\circ} \mathrm{C}$. Two sample sets reached temperatures of $6^{\circ} \mathrm{C}$ and $9^{\circ} \mathrm{C}$ due to a prolonged transportation. The background microbiota included 5.9 $\pm 0.2 \mathrm{log}$ CFU g ${ }^{-1}$ aerobic mesophiles, $5.0 \pm 0.3 \log$ CFU g ${ }^{-1}$ Enterobacteriaceae, and $<1.0 \log$ CFU g ${ }^{-1}$ E. coli.

\section{Results of the trial}

All inoculated samples were found positive for the corresponding STEC serogroup (Fig. 2), both for the low and the high inoculation level (Table 1). As a result, 100\% sensitivity was reached for the ring-trial. On the other hand, all noninoculated samples were reported negative, leading to $100 \%$ specificity of the ring-trial. As a consequence, both accordance and concordance of the ring-trial were $100 \%$.

Ten laboratories used both ready-to-use and in-house-prepared non-O157 agar plates, one laboratory used only readyto-use non-O157 agar plates, and one used only in-house-prepared non-O157 agar plates. Results did not differ between ready-to-use and in-house-prepared non-O157 agar plates.

Difficulties in the interpretation of the confirmation agar no. 3 containing D-arabinose (Fig. 1) were reported by different laboratories $(n=3)$. More specifically, serogroup O103, which were expected to be negative on the confirmation agar no. 3, and O111, which was expected positive on the confirmation agar no. 3, were both reported negative by one laboratory and were both reported positive by one laboratory; one laboratory could not interpret the results of this agar plate for both serogroups.

\section{Discussion}

All 12 participating laboratories in the international ringtrial successfully isolated both low (30 CFU $25 \mathrm{~g}^{-1}$ ) and high 
Table 1. Recovery of Low (25-33 CFU 25 G ${ }^{-1}$ ) ANd High (264-411 CFU 25 g $^{-1}$ ) Numbers OF Cold-STRESSED Shiga Toxin-Producing Escherichia coli (STEC) O26, O103, O111, and O145 from Artificially Contaminated Minced Beef by 12 Laboratories Participating in the International Ring-Trial

\begin{tabular}{|c|c|c|c|c|c|c|c|c|c|}
\hline \multirow[b]{3}{*}{ Laboratory } & \multirow{3}{*}{$\begin{array}{c}\text { Blank reference } \\
\text { (expected negative) }\end{array}$} & \multicolumn{8}{|c|}{ Inocula (CFU $25 g^{-1}$ ) } \\
\hline & & \multicolumn{2}{|c|}{ STEC O26 } & \multicolumn{2}{|c|}{ STEC O103 } & \multicolumn{2}{|c|}{ STEC 0111} & \multicolumn{2}{|c|}{ STEC 0145} \\
\hline & & 33 & 411 & 30 & 324 & 25 & 264 & 30 & 312 \\
\hline $\mathrm{C}$ & $0 / 2^{\mathrm{a}}$ & $2 / 2^{\mathrm{a}}$ & $2 / 2$ & $2 / 2$ & $2 / 2$ & $2 / 2$ & $2 / 2$ & $2 / 2$ & $2 / 2$ \\
\hline $\mathrm{D}$ & $0 / 2$ & $2 / 2$ & $2 / 2$ & $2 / 2$ & $2 / 2$ & $2 / 2$ & $2 / 2$ & $2 / 2$ & $2 / 2$ \\
\hline $\mathrm{E}$ & $0 / 2$ & $2 / 2$ & $2 / 2$ & $2 / 2$ & $2 / 2$ & $2 / 2$ & $2 / 2$ & $2 / 2$ & $2 / 2$ \\
\hline $\mathrm{F}$ & $0 / 2$ & $2 / 2$ & $2 / 2$ & $2 / 2$ & $2 / 2$ & $2 / 2$ & $2 / 2$ & $2 / 2$ & $2 / 2$ \\
\hline G & $0 / 2$ & $2 / 2$ & $2 / 2$ & $2 / 2$ & $2 / 2$ & $2 / 2$ & $2 / 2$ & $2 / 2$ & $2 / 2$ \\
\hline $\mathrm{H}$ & $0 / 2$ & $2 / 2$ & $2 / 2$ & $2 / 2$ & $2 / 2$ & $2 / 2$ & $2 / 2$ & $2 / 2$ & $2 / 2$ \\
\hline I & $0 / 2$ & $2 / 2$ & $2 / 2$ & $2 / 2$ & $2 / 2$ & $2 / 2$ & $2 / 2$ & $2 / 2$ & $2 / 2$ \\
\hline $\mathrm{J}$ & $0 / 2$ & $2 / 2$ & $2 / 2$ & $2 / 2$ & $2 / 2$ & $2 / 2$ & $2 / 2$ & $2 / 2$ & $2 / 2$ \\
\hline $\mathrm{K}$ & $0 / 2$ & $2 / 2$ & $2 / 2$ & $2 / 2$ & $2 / 2$ & $2 / 2$ & $2 / 2$ & $2 / 2$ & $2 / 2$ \\
\hline $\mathrm{L}$ & $0 / 2$ & $2 / 2$ & $2 / 2$ & $2 / 2$ & $2 / 2$ & $2 / 2$ & $2 / 2$ & $2 / 2$ & $2 / 2$ \\
\hline M & $0 / 2$ & $2 / 2$ & $2 / 2$ & $2 / 2$ & $2 / 2$ & $2 / 2$ & $2 / 2$ & $2 / 2$ & $2 / 2$ \\
\hline $\mathrm{N}$ & $0 / 2$ & $2 / 2$ & $2 / 2$ & $2 / 2$ & $2 / 2$ & $2 / 2$ & $2 / 2$ & $2 / 2$ & $2 / 2$ \\
\hline
\end{tabular}

${ }^{a}$ Number of samples with the expected result per number of samples tested.

All laboratories used both in-house-prepared and ready-to-use non-O157 agar plates, except laboratory G, which used only ready-to-use non-O157 agar plates, and laboratory N, which used only in-house-prepared non-O157 agar plates.

(300 CFU $25 \mathrm{~g}^{-1}$ ) levels of cold-stressed STEC O26, O103, O111, and O145 artificially contaminated into minced beef based on a selective enrichment followed by a selective isolation and confirmation on a non-O157 agar and confirmation agar plates described by Possé et al. (2008a), with subsequent serology testing. All non-inoculated samples were reported negative. Eleven out of the 12 laboratories used in-house-prepared non-O157 agar plates. Therefore, it was demonstrated that the method is applicable throughout different laboratories, including the preparation of all media. The current method aimed at isolating the four most important clinical non-O157 serogroups (O26, O103, O111, O145) on a single non-O157 agar plate (Possé et al., 2008a,b). This is a large improvement as isolation methods previously described target only one or two non-O157 STEC serogroups; for instance, an isolation method described by Hara-Kudo et al. (2002) specifically targeted O26, and an isolation method described by Safarikova and Safarik (2001) specifically targeted O26 and O111. Furthermore, a serogroup indication was provided directly on the plate by interpretation of the colony colors of the isolate on the nonO157 agar and confirmation agar plates. As a result, isolation as well as identification is achieved by a classical culture method combined with latex agglutination serology, with the advantage of directly obtaining the STEC isolate and the exclusion of molecular PCR steps. During the current ringtrial, food preservation conditions were simulated when preparing the artificially contaminated samples in order to validate the method for food analysis. To our knowledge, this is the first non-commercial isolation method for nonO157 STEC in food that is validated by an international ringtrial.

The numbers of aerobic mesophiles and Enterobacteriaceae were reported similar for all laboratories. Therefore, all laboratory results could be included in the study, notwithstanding the excessive duration of the shipment and the sample temperature at receipt (up to $9^{\circ} \mathrm{C}$ ) for two laboratories. The only problem of the method reported in the ring-trial was that the confirmation agar no. 3 containing D-arabinose was found difficult to interpret. However, serogroups $\mathrm{O} 103$ and O111, which both produce blue colonies on the non-O157 agar, can be differentiated on the basis of agar no. 2 alone, as these serogroups also differ in the ability to ferment dulcitol. As a consequence, the confirmation agar no. 3 containing $\mathrm{D}$-arabinose can be eliminated from the protocol.

In a previous study, the high sensitivity of the current detection and isolation method had already been demonstrated, as low levels (approximately $10 \mathrm{CFU} 25 \mathrm{~g}^{-1}$ ) of coldstressed STEC O26, O103, O111, and O145 were successfully isolated from minced beef (Verstraete et al., 2012). Similar detection limits were described in the literature for STEC $\mathrm{O} 26, \mathrm{O} 103, \mathrm{O} 111$, and $\mathrm{O} 145$ isolation from food (Hara-Kudo et al., 2002; Perelle et al., 2004; Possé et al., 2008b). In addition to its application for a wide range of sample matrices, varying from food (Verstraete et al., 2012) to cattle feces (Verstraete et al., 2010), this method was shown to be applicable by different laboratories as a result of our international ring-trial.

\section{Acknowledgments}

We thank Annelies Wachtelaer for her excellent technical assistance and Miriam Levenson for the English language editing of this manuscript. This research was funded by the Belgian Science Policy grant STECTRACK SD/AF/06A.

We acknowledge the following collaborators: F. Auvray (Anses [Agence Nationale de Sécurité Sanitaire], MaisonsAlfort, France); J. Boel (National Food Institute, Technical University of Denmark, Copenhagen, Denmark); N. Botteldoorn (Scientific Institute of Public Health, Brussels, Belgium); M. Cudjoe (National Veterinary Institute, Oslo, Norway); G. Daube (University of Liège, Liège, Belgium); K. De Reu (Institute for Agricultural and Fisheries Research, Melle, Belgium); L. De Zutter (Ghent University, Merelbeke, 
Belgium); A. Heuvelink (Food and Consumer Product Safety Authority, Zutphen, The Netherlands); D. Piérard (Universitair Ziekenhuis Vrije Universiteit Brussel, Brussels, Belgium); M. Rannou (ADRIA Development, Quimper, France); C. Rozand (Ecole Vétérinaire de Lyon, Lyon, France); and M. Uyttendaele (Ghent University, Ghent, Belgium).

\section{Disclosure Statement}

No competing financial interests exist.

\section{References}

Bettelheim KA. The non-O157 Shiga-toxigenic (verocytotoxigenic) Escherichia coli; under-rated pathogens. Crit Rev Microbiol 2007;33:67-87.

Beutin L. Emerging enterohaemorrhagic Escherichia coli, causes and effects of the rise of a human pathogen. J Vet Med B Infect Dis Vet Public Health 2006;53:299-305.

[EFSA] European Food Safety Authority. Technical specifications for the monitoring and reporting of verotoxigenic Escherichia coli (VTEC) on animals and food (VTEC surveys on animals and food). EFSA J 2009;7:1-43.

[EFSA] European Food Safety Authority. The European Union summary report on trends and sources of zoonoses, zoonotic agents and food-borne outbreaks in 2009. EFSA J 2011;9:200208.

Ethelberg S, Smith B, Torpdahl M, Lisby M, Boel J, Jensen T, Nielsen EM, Molbak K. Outbreak of non-O157 Shiga toxinproducing Escherichia coli infection from consumption of beef sausage. Clin Infect Dis 2009;48:e78-e81.

Hara-Kudo Y, Ikedo M, Komatsu O, Yamamoto S, Kumagai S. Evaluation of a chromogenic agar medium for isolation of Escherichia coli O26. Food Control 2002;13:377-379.

[ISO] International Organisation for Standardization. Microbiology of food and animal feeding stuffs. Protocol for the validation of alternative methods (ISO 16140:2003). Paris: European Committee for Standardization, AFNOR, 2003.

Karmali MA, Mascarenhas M, Shen S, Ziebell K, Johnson S, Reid-Smith R, Isaac-Renton J, Clark C, Rahn K, Kaper JB. Association of genomic O island 122 of Escherichia coli EDL 933 with verocytotoxin-producing Escherichia coli seropathotypes that are linked to epidemic and/or serious disease. J Clin Microbiol 2003;41:4930-4940.
Perelle S, Dilasser F, Grout JL, Fach P. Detection by $5^{\prime}$-nuclease PCR of Shiga toxin-producing Escherichia coli O26, O55, O91, O103, O111, O113, O145 and O157:H7, associated with the world's most frequent clinical cases. Mol Cell Probes 2004; 18:185-192.

Possé B, De Zutter L, Heyndrickx M, Herman L. Novel differential and confirmation plating media for Shiga toxinproducing Escherichia coli serotypes O26, O103, O111, O145 and sorbitol-positive and -negative O157. FEMS Microbiol Lett 2008a;282:124-131.

Possé B, De Zutter L, Heyndrickx M, Herman L. Quantitative isolation efficiency of O26, O103, O111, O145 and O157 STEC serotypes from artificially contaminated food and cattle faeces samples using a new isolation protocol. J Appl Microbiol 2008b;105:227-235.

Safarikova M, Safarik I. Immunomagnetic separation of Escherichia coli O26, O111 and O157 from vegetables. Lett Appl Microbiol 2001;33:36-39.

Schimmer B, Nygard K, Eriksen HM, Lassen J, Lindstedt BA, Brandal LT, Kapperud G, Aavitsland P. Outbreak of haemolytic uraemic syndrome in Norway caused by stx2-positive Escherichia coli O103:H25 traced to cured mutton sausages. BMC Infect Dis 2008;8.

Verstraete K, Robyn J, Del-Favero J, De Rijk P, Joris A, Herman L, Heyndrickx M, De Zutter L, De Reu K. Evaluation of a multiplex-PCR detection in combination with an isolation method for STEC O26, O103, O111, O145 and sorbitol fermenting O157 in food. Food Microbiol 2012;29:49-55.

Verstraete K, De Zutter L, Messens W, Herman L, Heyndrickx M, De Reu K. Effect of the enrichment time and immunomagnetic separation on the detection of Shiga toxin-producing Escherichia coli O26, O103, O111, O145 and sorbitol positive O157 from artificially inoculated cattle faeces. Vet Microbiol 2010;145:106-112.

Address correspondence to: Karen Verstraete, Ph.D. Technology and Food Science Unit Institute for Agricultural and Fisheries Research Brusselsesteenweg 370 9090 Melle, Belgium

E-mail: Koen.Dereu@ilvo.vlaanderen.be 